\title{
ANALYSIS OF A PARTIAL DECORRELATOR IN A MULTI-CELL DS/CDMA SYSTEM
}

\author{
Mohammad Saquib \\ ECE Department, LSU \\ Baton Rouge, LA 70803-5901 \\ e-mail:saquib@winlab.rutgers.edu
}

\author{
Roy Yates \\ WINLAB, Rutgers University \\ Piscataway NJ 08854-8060 \\ e-mail: ryates@winlab.rutgers.edu
}

\begin{abstract}
For a multi-cell CDMA system, we propose a partial decorrelator that decodes a user by suppressing the in-cell interferers only. As a result, each user suffers only from inter-cell interference and enhanced receiver noise. By analysis, we show that in random CDMA systems the partial decorrelator outperforms the conventional receiver, within the operating regime of the conventional receiver. By simulation, we show that under perfect power control, the partial decorrelator yields roughly 1.5 times the capacity of the conventional system.
\end{abstract}

\section{Introduction}

Although the decorrelator [1] has probably drawn more attention than any other multi-user detector, almost all studies have been for a single cell CDMA system. In a CDMA system with multiple cells all using the same frequency carrier, the implementation of a decorrelating detector and its performance are not well-understood. In a multi-cell environment, it is difficult for a base station to form the cross-correlation matrix by acquiring the signature and timing of all users in other cells. Moreover, the decorrelator exists only when the number of users is less than the processing gain. Thus it may not be possible to implement a true decorrelator in a multi-cell system with many users. For this environment, we propose a partial decorrelator, $(P D)$ that decodes a user by decorrelating the in-cell interferers only. With a PD, each user will suffer from other-cell interference but the implementation of the PD requires the knowledge of in-cell signatures only.

Similar to the current IS-95 DS/CDMA system, we adopt an R-CDMA system model in which different bits of a user are transmitted with random signature waveforms. We also assume that the time offset of a user is fixed throughout its transmission. With these assumptions, we compare the PD and the conventional receiver for an AWGN asynchronous multi-cell CDMA system.

In a single cell system when the processing gain is very large and the number of users is less than the processing gain, [4] shows that under both the conventional receiver and the decorrelator, the expected value of the signal-to-interference ratio (SIR) approaches the ratio of the average signal power to the average total interference power, which we call average SIR. Since, the bit error rate (BER) is difficult to ana- lyze for both the PD and the conventional receiver, average SIR is used in the analysis as a system performance measure. We verify by simulation that average SIR is a reliable performance measure for comparing the performance of the PD and the conventional receiver.

\section{System Model}

In our system model, each bit results in the baseband transmission of a sequence of pulses, $p[t]$. Each pulse has a duration of one chip period $T_{c}$. These pulses are sent over an additive white Gaussian noise channel in which the noise $N(t)$ has power spectral density $\sigma^{2}$.

We assume there are $K$ users in the system with the in-cell users numbered 1 through $K_{1}$ and the other-cell users enumerated from $K_{1}+1$ to $K$. The bit transmission time of a user is $T$ and the processing gain is $L=T / T_{c}$. To transmit the $i$ th bit, the $j$ th user employs the following signature waveform.

$$
s_{j}^{(i)}(t)=\sum_{m=1}^{L} A_{j}^{(i)}(m) \frac{1}{\sqrt{L}} p\left[t-(m-1) T_{c}\right]
$$

where $A_{j}^{(i)}(m) \in\{-1,1\}$ denotes the signature sequence of user $j$ which is used for transmitting the $i$ th bit. The energy of the pulse $p[t]$ is normalized so that $\int_{0}^{T}\left[s_{j}^{(i)}(t)\right]^{2} d t=1$ for all $j$. Let $\Delta_{j}$ denote the delay of the $j$ th user. In the asynchronous channel, the received signal due to the $j$ th user at the desired user's base station is

$$
r_{j}(t)=\sum_{i=-\infty}^{+\infty} \sqrt{E_{j}} b_{j}^{(i)} s_{j}^{(i)}\left(t-i T-\Delta_{j}\right)
$$

where $b_{j}^{(i)} \in\{-1,+1\}$ is the $i$ th bit and $E_{j}$ is the received energy of the $j$ th user at the desired user's base station. We model the bits and signature sequences as independent, identically distributed (i.i.d) equally likely binary sequences. From the total received signal,

$$
R(t)=\sum_{j=1}^{K} r_{j}(t)+N(t)
$$

we wish to decode bit 0 of user $1, b_{1}^{(0)}$, assuming $\Delta_{1}=0$. 


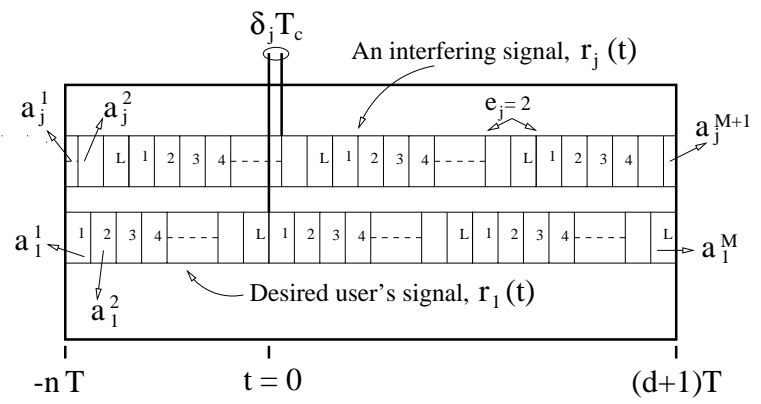

Figure 1: This figure depicts the normalized chip waveforms $\left\{a_{j}^{i}\right\}$ of the received signal $r_{j}(t)$ of equation (2) for $t \in[-n T,(d+1) T]$.

\section{Chip Matched Filter}

We apply chip matched filtering to implement both the conventional receiver and the PD. The received signal $R(t)$ of equation (3) is sent through a chip matched filter and sampled at the chip rate. The bit $b_{1}^{(0)}$ will be processed by employing an observation window of length $n+d+1$ bits, where $n$ is the number of bits into the past and $d$ is the number of bits into the future. The vector of chip matched filter output samples in $[-n T,(d+1) T]$ is $\mathbf{R}=\left[R_{1}, \cdots, R_{M}\right]^{\top}$ where $M=(n+d+1) L$. For a rectangular chip waveform, the $m$ th chip sample is

$$
R_{m}=\int_{-n T+(m-1) T_{c}}^{-n T+m T_{c}} r(t) d t \quad m=1, \ldots, M
$$

In equation (4), $R_{m}$ is a function of the parameters of the asynchronous DS/CDMA system. In the observation window $[-n T,(d+1) T]$, an interfering user $j$ transmits $M+1$ chips or partial chips while user 1 transmits exactly $M$ chips. For notational convenience, we denote by $a_{j}^{m}$ the $m$ th chip of user $j$ in the observation window $[-n T,(d+1) T]$. The chips $a_{j}^{m}$ are depicted Figure 1 where it is shown that for $j \neq 1$, chips $a_{j}^{1}$ and $a_{j}^{M+1}$ are truncated at the left and right boundaries of the observation window. To model an R-DS/CDMA system, we assume $\left\{a_{j}^{m}\right\}$ is an i.i.d equally likely $\pm 1 / \sqrt{L}$ sequence.

Since all users transmit asynchronously, during the observation window $[-n T,(d+1) T]$, user 1 transmits the $(n+$ $d+1)$ bits, $\left\{b_{1}^{(i)} \mid i=-n, \ldots, d\right\}$, while an interfering user $j$ transmits the $(n+d+2)$ bits $\left\{b_{j}^{(i)} \mid i=-n-1, \ldots, d\right\}$. Among those $(n+d+2)$ interfering bits, $b_{j}^{(-n-1)}$ and $b_{j}^{(d)}$ are partial bits which are truncated at the left boundary $-n T$ and the right boundary $(d+1) T$ respectively. We can write the total received signal as $\mathbf{R}=\sum_{j=1}^{K} \mathbf{r}_{j}+\mathbf{N}$ where

$$
\mathbf{r}_{j}=\sum_{i=-n-1}^{d} b_{j}^{(i)} \sqrt{E_{j}} \overline{\mathbf{S}}_{j}^{(i)} \quad j \neq 1
$$

is the contribution of the $j$ th interfering user and $\mathbf{N}$ is an $M \times$ 1 Gaussian noise vector with cross-correlation matrix $\sigma^{2} T_{c} \mathbf{I}$. Note that $\overline{\mathbf{S}}_{j}^{(i)}$ is an $M \times 1$ vector that represents an effective chip waveform for bit $i$ of user $j$ over the observation window.

We will now characterize $\overline{\mathbf{S}}_{j}^{(i)}$ in terms of the users' delays $\Delta_{j}$. Without loss of generality, we can assume $\Delta_{j} \in$ $[0, T)$, and we can write $\Delta_{j}=\left(e_{j}+\delta_{j}\right) T_{c}$, where $e_{j} \in$ $\{0,1, \ldots, L-1\}$ and $\delta_{j} \in[0,1)$. In addition, we adopt the notation $\mathbf{O}_{k}$ for a $k$ element row vector of zeros, $\bar{\delta}_{j}=1-\delta_{j}$, and the row vector $\mathbf{a}_{j}(l, m)=\left[a_{j}^{l}, \ldots, a_{j}^{m}\right]$ in order to express $\overline{\mathbf{S}}_{j}^{(i)}$ as follows.

1. Consider $i=-n-1$. If $e_{j}=0$, then

$$
\overline{\mathbf{S}}_{j}^{(i)}=\delta_{j}\left[\mathbf{a}_{j}(1,1), \mathbf{O}_{M-1}\right]^{\top}
$$

If $e_{j}>0$, then let $\bar{e}_{j}=e_{j}+1$ and $\bar{M}=M-e_{j}$ and

$$
\overline{\mathbf{S}}_{j}^{(i)}=\delta_{j}\left[\mathbf{a}_{j}\left(1, \bar{e}_{j}\right), \mathbf{O}_{\bar{M}-1}\right]^{\top}+\bar{\delta}_{j}\left[\mathbf{a}_{j}\left(2, \bar{e}_{j}\right), \mathbf{O}_{\bar{M}}\right]^{\top}
$$

2. When $i=-n+x$ for $x \in\{0, \ldots, n+d-1\}$, let $V_{x}=e_{j}+x L$ and $W_{x}=M-e_{j}-(x+1) L$ so that

$$
\begin{aligned}
\overline{\mathbf{S}}_{j}^{(i)}= & \bar{\delta}_{j}\left[\mathbf{O}_{V_{x}}, \mathbf{a}_{j}\left(\hat{V}_{x}, \hat{V}_{x}\right), \mathbf{O}_{W_{x}}\right]^{\top} \\
& +\delta_{j}\left[\mathbf{O}_{V_{x}+1}, \mathbf{a}_{j}\left(V_{x}+2, \bar{V}_{x}+L\right), \mathbf{O}_{W_{x}-1}\right]^{\top}
\end{aligned}
$$

3. When $i=d$, we define $H=(n+d) L+e_{j}+1$ and

$$
\begin{aligned}
\overline{\mathbf{S}}_{j}^{(i)}=\bar{\delta}_{j}\left[\mathbf{O}_{H-1}, \mathbf{a}_{j}(H+1, M+1)\right]^{\top} \\
+\delta_{j}\left[\mathbf{O}_{H}, \mathbf{a}_{j}(H+1, M)\right]^{\top}
\end{aligned}
$$

For user 1, we have $e_{1}=\tau_{1}=0$ and as a consequence,

$$
\mathbf{r}_{1}=b_{1}^{(0)} \sqrt{E_{1}} \mathbf{S}_{1}^{(0)}+\overline{\mathbf{r}}_{1}
$$

where the in-cell interference from user 1's other bits is

$$
\overline{\mathbf{r}}_{1}=\sum_{i=-n}^{-1} b_{1}^{(i)} \sqrt{E_{1}} \mathbf{S}_{1}^{(i)}+\sum_{i=1}^{d} b_{1}^{(i)} \sqrt{E_{1}} \mathbf{S}_{1}^{(i)}
$$

Furthermore, we can write

$$
\mathbf{S}_{1}^{(i)}=\left[\mathbf{O}_{v_{i}}, \mathbf{a}_{1}\left(v_{i}+1, v_{i}+L\right), \mathbf{O}_{M-v_{i}-L}\right]^{\top}
$$

where $v_{i}=(n+i) L$. The total received signal vector $\mathbf{R}$ is the sum of the desired signal $\mathbf{R}_{d}$, the intra-cell interference $\mathbf{R}_{i}$, and the other-cell interference $\mathbf{R}_{o}$. That is,

$$
\mathbf{R}=\sum_{j=1}^{K} \mathbf{r}_{j}+\mathbf{N}=\mathbf{R}_{d}+\mathbf{R}_{i}+\mathbf{R}_{o}+\mathbf{N}
$$

Note that $\mathbf{R}_{d}=b_{1}^{(0)} \sqrt{E_{1}} \mathbf{S}_{1}^{(0)}$ while $\mathbf{R}_{i}=\overline{\mathbf{r}}_{1}+\sum_{j=2}^{K_{1}} \mathbf{r}_{j}$. Lastly, the other-cell interference is $\mathbf{R}_{o}=\sum_{j=K_{1}+1}^{K} \mathbf{r}_{j}$. 


\section{Performance Comparison}

For bit $b_{1}^{(0)}$, the conventional receiver output is

$$
R^{\mathrm{cd}}=\mathbf{S}_{1}^{(0)^{\top}} \mathbf{R}=\sqrt{E_{1}} b_{1}^{(0)}+R_{i}^{\mathrm{cd}}+R_{o}^{\mathrm{cd}}+n^{\mathrm{cd}}
$$

In equation (12), $n^{\text {cd }}$ is a Gaussian random variable with mean zero and variance $\sigma_{0}^{2}=\sigma^{2} T_{c}$. The term $R_{i}^{\text {cd }}=\left(\mathbf{S}_{1}^{(0)}\right)^{\top} \mathbf{R}_{i}$ denotes the in-cell interference and $R_{o}^{\text {cd }}=\left(\mathbf{S}_{1}^{(0)}\right)^{\top} \mathbf{R}_{o}$ is the other-cell interference. We denote the second moments of the in-cell and other-cell interference by

$$
\sigma_{1}^{2}=\mathrm{E}\left[\left(R_{i}^{\mathrm{cd}}\right)^{2}\right] \quad \sigma_{2}^{2}=\mathrm{E}\left[\left(R_{o}^{\mathrm{cd}}\right)^{2}\right]
$$

Note that $\sigma_{1}^{2}$ and $\sigma_{2}^{2}$ represent the average in-cell and othercell interference power observed at the output of the conventional receiver of user 1 .

Ignoring the other-cell interference, we follow the working principles of the asynchronous multi-rate decorrelator (AMD) of reference [3] to implement the PD. In the other words, while treating the multiuser interference from other cells as a background noise, the PD decodes $b_{1}^{(0)}$ by orthogonalizing all the in-cell interfering signatures within the observation window $[-n T,(d+1) T]$. Over this window, we use $\mathbf{S}_{n, d}$ to denote the set of in-cell interfering signatures. Let $\boldsymbol{\Phi}_{1}$ be the PD filter that decodes $b_{1}^{(0)}$. Reference [3] finds $\boldsymbol{\Phi}_{1}$ by applying Gram-Schmidt orthogonalization on the interfering users' signature sequences $\mathbf{S}_{n, d}$. When $\mathbf{S}_{1}^{(0)}$ is a linear combination of the signatures in $\mathbf{S}_{n, d}, \boldsymbol{\Phi}_{1}=0$. Let $A$ be the event that $\mathbf{S}_{1}^{(0)}$ is linearly independent of $\mathbf{S}_{l} \in \mathbf{S}_{n, d}$. We use $A^{c}$ to denote the complement.

Let us first consider the non-trivial event $A$. In this case, the output of $\boldsymbol{\Phi}_{1}$ will be

$$
R^{\mathrm{pd}}=\mathbf{\Phi}_{1}^{\top} \mathbf{r}=\sqrt{\zeta_{n, d} E_{1}} b_{1}^{(0)}+R_{o}^{\mathrm{pd}}+n^{\mathrm{pd}}
$$

where $n^{\text {pd }}$ is a Gaussian random variable with mean zero and variance $\hat{\sigma}_{0}^{2}=\sigma^{2} T_{c}$. The term $R_{o}^{\mathrm{pd}}=\boldsymbol{\Phi}_{1}^{\top} \mathbf{R}_{o}$ denotes the other-cell interference and $\zeta_{n, d}=\left(\boldsymbol{\Phi}_{1}^{\top} \mathbf{S}_{1}^{(0)}\right)^{2}$ is the asymptotic efficiency [1] of the PD for decoding the bit $b_{1}^{(0)}$ when $\mathbf{R}_{o}=0$. Note that when the event $A^{c}$ occurs, $\boldsymbol{\Phi}_{1}=0$ and hence $R^{\text {pd }}=0$. In this case, the AWGN variance at the PD output will be trivially zero. Otherwise, the AWGN variance $\hat{\sigma}_{0}^{2}$ at the PD output will equal $\sigma_{0}^{2}$. In either case, we have $\hat{\sigma}_{0}^{2} \leq \sigma_{0}^{2}$. We denote the second moment of $R_{o}^{\mathrm{pd}}$ as $\sigma_{3}^{2}$. The term $\sigma_{3}^{2}$ denotes the average other-cell interfering powers observed by user 1 under the PD system.

If $\mathrm{SIR}_{\mathrm{cd}}$ and $\mathrm{SIR}_{\mathrm{pd}}$ denote average SIR of user 1 under the conventional detector and the PD system respectively, then

$$
\operatorname{SIR}_{\mathrm{cd}}=\frac{E_{1}}{\sigma_{1}^{2}+\sigma_{2}^{2}+\sigma_{0}^{2}} \quad \operatorname{SIR}_{\mathrm{pd}}=\frac{\mathrm{E}\left[\zeta_{n, d}\right] E_{1}}{\sigma_{3}^{2}+\hat{\sigma}_{0}^{2}}
$$

Let us denote the ratio $\left(\sigma_{2}^{2}+\sigma_{0}^{2}\right) / \sigma_{1}^{2}$ as $F$. In a conventional multi-cell DS/CDMA system, $\sigma_{2}^{2} / \sigma_{1}^{2} \approx 0.55$ [5]. This fact suggests that the parameter $F$ is a constant and in an interference limited system, its value would be approximately 0.55 .

Our goal is to compare the capacity of the PD with that of the conventional receiver. Therefore, we would like to develop an upper bound on the number of in-cell users, $K_{1}$ as a function of function system parameters for which $\operatorname{SIR}_{\mathrm{cd}} \leq$ $\mathrm{SIR}_{\mathrm{pd}}$. To develop the desired upper bound on $K_{1}$, we need to prove the following two lemmas.

Lemma 1 In $R$-CDMA systems, for $n, d \geq 0$,

$$
E\left[\zeta_{n, d}\right] \geq E\left[\zeta_{0,0}\right] \geq 1-2\left(K_{1}-1\right) / L
$$

Proof: Reference [3] proves that $\zeta_{n, d}$ is monotonic increasing function of $n, d$, so that for $n, d \geq 0, \zeta_{n, d} \geq \zeta_{0,0}$. Note that $\zeta_{0,0}$ is the asymptotic efficiency [1] of the one-shot decorrelator operating with the observation window $[0, T]$ and zero inter-cell interference. Within the observation window $[0, T]$, $b_{1}^{(0)}$ experiences interference from two bits of an interfering user $j$. As a consequence, the number of elements of $\mathbf{S}_{n, d}$ for the observation window $[0, T]$ is $2\left(K_{1}-1\right)$. Let $2\left(K_{1}-1\right)$ incell interfering signatures of size $L \times 1$ generate $K_{1}^{\prime}$ orthonormal basis vectors $\left\{\mathbf{F}_{j}\right\}$. Since $\mathbf{S}_{l} \in \mathbf{S}_{n, d}$ consists of random signatures $\left\{a_{j}^{i}\right\}, K_{1}^{\prime}$ will be a random variable. Furthermore, for given $\mathbf{S}_{n, d}$, if all $\mathbf{S}_{l} \in \mathbf{S}_{n, d}$ are linearly independent, then $K_{1}^{\prime}=2\left(K_{1}-1\right)$, otherwise, $K_{1}^{\prime}<2\left(K_{1}-1\right)$. Since in either case $K_{1}^{\prime} \leq 2\left(K_{1}-1\right)$, we have $\mathrm{E}\left[K_{1}^{\prime}\right] \leq 2\left(K_{1}-1\right)$.

For given $\mathbf{S}_{n, d}$, we can write $\zeta_{0,0}$ as follows.

$$
\zeta_{0,0}=1-\sum_{j=1}^{K_{1}^{\prime}}\left(\mathbf{F}_{j}^{\top} \mathbf{S}_{1}^{(0)}\right)^{2}=1-\sum_{j=1}^{K_{1}^{\prime}} \mathbf{F}_{j}^{\top} \mathbf{S}_{1}^{(0)}\left(\mathbf{S}_{1}^{(0)}\right)^{\top} \mathbf{F}_{j}
$$

Since $\mathrm{E}\left[\mathbf{S}_{1}^{(0)} \mathbf{S}_{1}^{(0)^{\top}}\right]=(1 / L) \mathbf{I}_{L}$ and $\mathbf{F}_{j}^{\top} \mathbf{F}_{j}=1$, taking the expectation with respect to $\mathbf{S}_{1}^{(0)}$ on both sides of the above equation yields

$$
\mathrm{E}_{\mathbf{S}_{1}^{(0)}}\left[\zeta_{0,0}\right]=1-K_{1}^{\prime} / L
$$

We will now take expectation with respect to $K_{1}^{\prime}$ on both sides of equation (17). Since $\mathrm{E}\left[K_{1}^{\prime}\right] \leq 2\left(K_{1}-1\right)$, we will then get

$$
\mathrm{E}\left[\zeta_{0,0}\right]=1-\mathrm{E}\left[K_{1}^{\prime}\right] / L \geq 1-2\left(K_{1}-1\right) / L
$$

For all $n, d \geq 1, \zeta_{n, d} \geq \zeta_{0,0}$, implying $\mathrm{E}\left[\zeta_{n, d}\right] \geq \mathrm{E}\left[\zeta_{0,0}\right]$, from which the claim follows.

The near-far resistance [1] of the decorrelator is the same as its asymptotic efficiency and is equal to the near-far resistance of the LMMSE receiver. We observe that the lower bound (18) is the same as that for the average near-far resistance of the one 
shot LMMSE receiver derived in [2]. In addition, Lemma 1 shows that for $n, d \geq 0$, the lower bound of $\mathrm{E}\left[\zeta_{0,0}\right]$ is also a lower bound to $\mathrm{E}\left[\zeta_{n, d}\right]$. Now we will state the second lemma with proof.

Lemma 2 In chip synchronous $R$-CDMA systems, the second moment of the inter-cell interference is higher under the conventional system than the $P D$ system, i.e., $\sigma_{2}^{2} \geq \sigma_{3}^{2}$.

Proof: For the sake of simplicity, we use $\hat{\boldsymbol{\Phi}}_{1}$ to denote the receiver filter that is applied to $\mathbf{R}$ to decode $b_{1}^{(0)}$. That is, $\hat{\mathbf{\Phi}}_{1}=\mathbf{S}_{1}^{(0)}$ for the conventional detector or $\hat{\boldsymbol{\Phi}}_{1}=\boldsymbol{\Phi}_{1}$ for the partial decorrelator. If $\bar{R}_{o}$ is the other-cell interference observed at the desired receiver filter output, then equation (11) implies

$$
\bar{R}_{o}=\hat{\mathbf{\Phi}}_{1}^{\top} \mathbf{R}_{o}=\sum_{j=K_{1}+1}^{K} \hat{\mathbf{\Phi}}_{1}^{\top} \mathbf{r}_{j}
$$

Squaring equation (19), we obtain

$$
\bar{R}_{o}^{2}=\hat{\boldsymbol{\Phi}}_{1}^{\top} \mathbf{R}_{o} \mathbf{R}_{o}^{\top} \hat{\boldsymbol{\Phi}}_{1}=\sum_{j=K_{1}+1}^{K} \sum_{k=K_{1}+1}^{K} \hat{\boldsymbol{\Phi}}_{1}^{\top} \mathbf{r}_{j} \mathbf{r}_{k}^{\top} \hat{\boldsymbol{\Phi}}_{1}
$$

Using equation (5) with equation (20), we write

$$
\bar{R}_{o}^{2}=\sum_{j=K_{1}+1}^{K} \sum_{k=K_{1}+1}^{K} \sum_{l=-n-1}^{d} \sum_{i=-n-1}^{d} \hat{r}(i, l, j, k)
$$

where

$$
\hat{r}(i, l, j, k)=b_{j}^{(l)} b_{k}^{(i)} \sqrt{E_{j}^{(l)}} \sqrt{E_{k}^{(i)}} \hat{\mathbf{\Phi}}_{1}^{\top} \overline{\mathbf{S}}_{j}^{(l)}\left(\overline{\mathbf{S}}_{k}^{(i)}\right)^{\top} \hat{\mathbf{\Phi}}_{1}
$$

Since the transmitted bits are an iid equally likely \pm 1 sequence, taking the expectation with respect to transmitted bits on both sides of the above equation, we obtain

$$
\mathrm{E}_{b}\left(\bar{R}_{o}^{2}\right)=\sum_{j=K_{1}+1}^{K} \sum_{i=-n-1}^{d} E_{j}^{(i)} \hat{\mathbf{\Phi}}_{1}^{\top} \overline{\mathbf{S}}_{j}^{(i)}\left(\overline{\mathbf{S}}_{j}^{(i)}\right)^{\top} \hat{\mathbf{\Phi}}_{1}
$$

Recall that when the event $A$ occurs, for the partial decorrelator, $\left\|\hat{\boldsymbol{\Phi}}_{1}\right\|^{2}=1$, otherwise, $\left\|\hat{\boldsymbol{\Phi}}_{1}\right\|^{2}=0$. However, for the conventional receiver, $\left\|\hat{\boldsymbol{\Phi}}_{1}\right\|^{2}=1$ always. We assume the event $A$ occurs with probability $\operatorname{Pr}[A]=p$. Let $\mathbf{C}_{M}$ be an $M \times M$ symmetric matrix whose $(l, m)$ th element is 1 if $|l-m|=1$ and 0 otherwise. To calculate the second moment of $\bar{R}_{o}$ given event $A$, we take the expectation with respect to signatures $\overline{\mathbf{S}}_{j}^{(i)}$. Note that

$$
\begin{aligned}
& \sum_{i=-n-1}^{d} \mathrm{E}_{\overline{\mathbf{S}}_{j}^{(i)}}\left[\overline{\mathbf{S}}_{j}^{(i)}\left(\overline{\mathbf{S}}_{j}^{(i)}\right)^{\top}\right] \\
& \quad=\frac{\left(1-\delta_{j}\right)^{2}+\delta_{j}^{2}}{L} \mathbf{I}_{M}+\frac{\delta_{j}\left(1-\delta_{j}\right)}{L} \mathbf{C}_{M}
\end{aligned}
$$

This implies

$$
\begin{aligned}
& \sum_{i=-n-1}^{d} \hat{\mathbf{\Phi}}_{1}^{\top} \mathrm{E}_{\overline{\mathbf{S}}_{j}^{(i)}}\left[\overline{\mathbf{S}}_{j}^{(i)}\left(\overline{\mathbf{S}}_{j}^{(i)}\right)^{\top}\right] \hat{\mathbf{\Phi}}_{1}= \\
& \quad \frac{\left(1-\delta_{j}\right)^{2}+\delta_{j}^{2}}{L} \hat{\boldsymbol{\Phi}}_{1}^{\top} \hat{\mathbf{\Phi}}_{1}+\frac{\delta_{j}\left(1-\delta_{j}\right)}{L} \hat{\boldsymbol{\Phi}}_{1}^{\top} \mathbf{C}_{M} \hat{\mathbf{\Phi}}_{1}
\end{aligned}
$$

Using $\left\|\hat{\boldsymbol{\Phi}}_{1}\right\|^{2}=1$, and $\delta_{j}=0$ with the above equation, for the chip synchronous system, we obtain the second moment of the inter-cell interference given the event $A$ as follows.

$$
\mathrm{E}\left[\bar{R}_{o}^{2} \mid A\right]=\mathrm{E}_{\overline{\mathbf{S}}_{j}^{(i)}}\left[\mathrm{E}_{b}\left(\bar{R}_{o}^{2}\right)\right]=\sum_{j=K_{1}+1}^{K} \frac{E_{j}}{L}
$$

We will now consider the event $A^{c}$. Given the event $A^{c}$, $\left\|\mathbf{\Phi}_{1}\right\|^{2}=0$ and for the PD, $\mathrm{E}\left[\bar{R}_{o}^{2} \mid A^{c}\right]$ is trivially zero. However, $\mathrm{E}\left[\bar{R}_{o}^{2} \mid A^{c}\right]$ for the conventional receiver is the same as equation (26). Combining these facts with equation (26), we obtain

$$
\sigma_{3}^{2}=p \mathrm{E}\left[\bar{R}_{o}^{2} \mid A\right] \leq \sum_{j=K_{1}+1}^{K} \frac{E_{j}}{L}=\sigma_{2}^{2}
$$

Applying the fact $\hat{\sigma}_{0}^{2} \leq \sigma_{0}^{2}$ with lemmas 1 and 2 in equation (15), we prove the following theorem.

Theorem 1 In chip synchronous $R-C D M A$ systems, $K_{1}<$ $L /[2(1+F)]+1$ implies that the $P D$ provides higher average SIR than the conventional receiver.

In an interference limited CDMA system, $F \approx 0.55$. In this case, when $K_{1} / L<0.3125$, the PD will be better. However, for conventional systems, $K_{1} / L \approx 0.2$. This suggests that within the operating regime of the conventional receiver, the PD will outperform the conventional receiver. Furthermore, note that Theorem 1 is based on the lower bound on $\mathrm{E}\left[\zeta_{0,0}\right]$ in Lemma 1. Since for $n, d>0, \mathrm{E}\left[\zeta_{n, d}\right] \geq \mathrm{E}\left[\zeta_{0,0}\right]$, one could expect the PD with $n, d \geq 1$ to outperform the conventional receiver even when the number of in-cell users will be greater than $L /[2(1+F)]+1$.

\section{Empirical Results}

To compare the performance of the PD with the conventional receiver, a simulation study was performed with an asynchronous multi-cell DS/CDMA system of seven contiguous hexagonal cells. The area of each cell was approximately $\pi 1000^{2}$ meters $^{2}$ which is the area of a circle of radius $r_{o}=1000$ meters. The cell which was in the middle of seven cells was the cell of interest. It was assumed that a mobile was uniformly distributed within its own cell. This assumption yielded a probability density function $f(r)=2 r / r_{0}^{2}$ for the distance of a user from its own base station. The number 


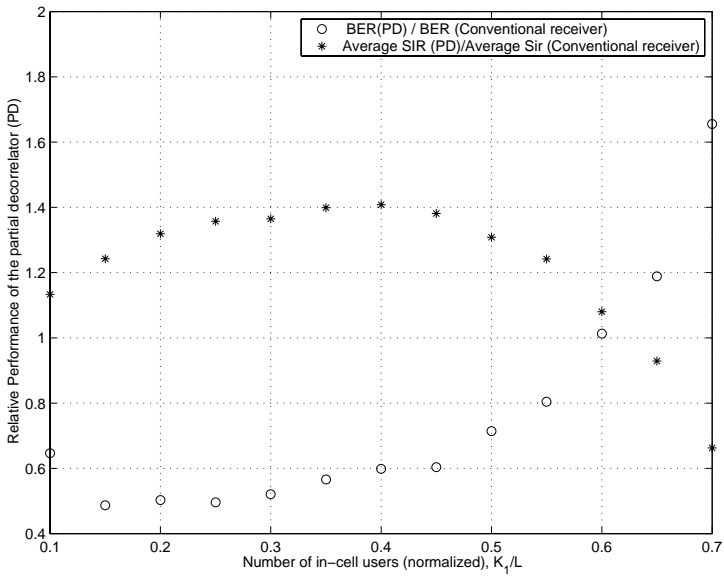

Figure 2: Relative performance of the PD is plotted as function of $K_{1} / L$.

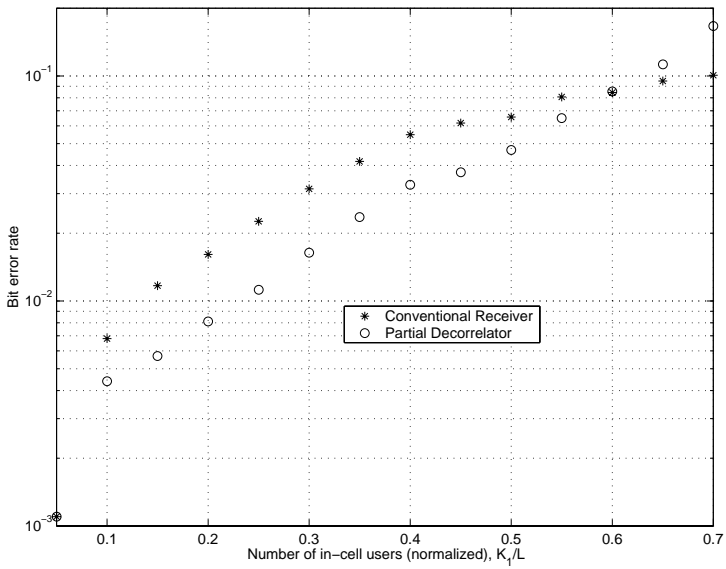

Figure 3: Bit error rate of user 1 is shown as a function of $K_{1} / L$ under the PD and the conventional systems.

of in-cell users is $1 / 7$ th of the total number of users in the system. We used a path loss exponent $=4$. The height of the base station was 30 meters so that the uplink channel gain of user $j$ to its own base station, $h_{j}$ was $1 /\left(r^{2}+30^{2}\right)^{2}$. The system processing gain was 20 and chips were asynchronous. Perfect power control was assumed, i.e., every user has the same received power at its own base station and its SNR was $9.8 \mathrm{~dB}$ which yields BER $10^{-3}$ in a single user channel. The observation window of the PD was $n=d=1$. In each iteration of our simulation, the received signal of equation (3) was passed through a chip matched filter and sampled at the chip rate. The sampled vector of length $(n+d+1) L=60$ was then processed by both the conventional receiver and the PD to recover the desired transmitted bit.

Recall that Lemma 2 claims $\sigma_{3}^{2} \leq \sigma_{2}^{2}$ for chip synchronous systems. A similar result was observed in our simulations for the chip asynchronous system. Space constraints force us to omit that result.

In a CDMA system, BER is the performance measure of interest. Since for the PD and the conventional receiver, BER is hard to analyze, in our analysis we use the average SIR (i.e., the ratio of the average signal power to the average interference power) as the system performance measure. Our simulation results showed that $\operatorname{SIR}_{\mathrm{cd}} \leq \mathrm{SIR}_{\mathrm{pd}}$ is equivalent to $\mathrm{BER}_{1}^{\mathrm{cd}} \geq \mathrm{BER}_{1}^{\mathrm{pd}}$, where $\mathrm{BER}_{1}^{\mathrm{cd}}$ is the BER of user 1 under the conventional system and $\mathrm{BER}_{1}^{\mathrm{pd}}$ is the BER of user 1 under the PD system; see Figure 2.

The BER requirement of a conventional system is approximately $10^{-2}$ which is obtained at $K_{1} / L \approx 0.2$. Our simulation result, also agreed with this previous observation; see Figure 3. Here, we also found that the PD's performance at $K_{1} / L=0.3$ is the same as the performance of the conventional receiver at $K_{1} / L=0.2$. This result suggests that the PD yields $50 \%$ capacity gain over the conventional receiver.

\section{References}

[1] R. Lupas and S. Verdú. Near-Far Resistance of Multiuser Detectors in Asynchronous Channels. IEEE Trans. On Comm., 38(4):496-508, April 1990.

[2] U. Madhow and M. L. Honig. On the average near-far resistance for MMSE detection of direct- sequence CDMA signals with random spreading,. IEEE Trans. on Info. Theory. (accepted).

[3] M. Saquib, R. Yates, and A. Ganti. An Asynchronous Decentralized Multi-rate Decorrelator. In Proc. of the CISS, volume 1, pages 462-467, Johns Hopkins University, USA, March, 19-21 1997.

[4] D. Tse and S. Hanly. Linear Multiuser Receivers: Effective Interference, Effective Bandwidth and User Capacity. IEEE Trans. on Info. Theory. (accepted).

[5] A. J. Viterbi. The Orthogonal-Random Waveform Dichotomy for Digital Mobile Personal Communications . IEEE Personal Comm., (First Quarters):18-24, 1994. 